\title{
MAGNETIC SUSCEPTIBILITY OF $\mathrm{Sn}_{1-x} \mathrm{Eu}_{x} \mathrm{Te}^{*}$
}

\author{
M. Górska ${ }^{a}$, J.R. ANDERSON ${ }^{b}$, J.L. Peng ${ }^{b}$ AND Z. GolaCKI ${ }^{a}$ \\ anstitute of Physics, Polish Academy of Sciences \\ Al. Lotników 32/46, 02-668 Warszawa, Poland \\ ${ }^{b}$ Department of Physics, University of Maryland, College Park, MD 20742, USA
}

The magnetic susceptibility of Bridgman-grown $\mathrm{Sn}_{1-x} \mathrm{Eu}_{x} \mathrm{Te}$ with nominal values of $x$ up to 0.095 was measured over a temperature range from 2 to $385 \mathrm{~K}$. The samples were $p$-type with hole concentrations up to $1.5 \times 10^{21} \mathrm{~cm}^{-3}$. The susceptibility data above $50 \mathrm{~K}$ followed the Curie-Weiss relation with a small Curie temperature. At about $10 \mathrm{~K}$ a small cusp in susceptibility was observed in samples with the higher range of $x$ values. The data indicate a presence of both antiferromagnetic and ferromagnetic exchange interactions, with some paramagnetic contributions from charged defects. There is also evidence of Eu going into the lattice both as $\mathrm{Eu}^{2+}$ and $\mathrm{Eu}^{3+}$ ions.

PACS numbers: $75.20 . \mathrm{Ck}, 75.30 . \mathrm{Et}$

\section{Introduction}

Magnetic properties of rare-earth-doped IV-VI chalcogenides have been studied recently in $\mathrm{Pb}_{1-x} \mathrm{Gd}_{x} \mathrm{Te}, \mathrm{Pb}_{1-x} \mathrm{Eu}_{x} \mathrm{Te}$, and $\mathrm{Sn}_{1-x} \mathrm{Gd}_{x} \mathrm{Te}$ [1-3]. The results showed a very weak antiferromagnetic exchange coupling among magnetic ions, with no evidence of RKKY-type ferromagnetic interaction observed in $\mathrm{Pb}_{1-x-y} \mathrm{Sn}_{y} \mathrm{Mn}_{x} \mathrm{Te}$ [4]. The susceptibility measurements of $\mathrm{Sn}_{1-x} \mathrm{Eu}_{x} \mathrm{Te}$ by Mathur et al. at temperatures up to $70 \mathrm{~K}$ showed a deviation from the Curie-Weiss behavior similar to that in spin-glass materials [5]. Here we are reporting results on $\mathrm{Sn}_{1-x} \mathrm{Eu}_{x} \mathrm{Te}$ at temperatures up to $385 \mathrm{~K}$.

\section{Experiment}

The samples of $\mathrm{Sn}_{1-x} \mathrm{Eu}_{x} \mathrm{Te}$ were cut from boules grown by the Bridgman technique. The $x_{\mathrm{v}}$ values determined by electron microprobe analysis and the hole concentrations at $4.2 \mathrm{~K}$ (both with an accuracy of about $20 \%$, including variation throughout the sample) are given in Table.

The magnetic susceptibility was measured using a SQUID detection system at temperatures from 2 to $385 \mathrm{~K}$ and at magnetic fields from 5 to 500 Oe. The SnTe host diamagnetic susceptibility was measured from 2 to $375 \mathrm{~K}$ at fields from 0.5 to $5 \mathrm{kOe}$. The experimental method and details were described previously $[2,3]$.

${ }^{*}$ This work was supported in part by the M. Skłodowska-Curie Joint Fund II, No. PAN/NSF-92-113 and by the Committee for Scientific Research grant No. 204829101. 


\section{TABLE}

Susceptibility fitting parameters and carrier concentrations.

\begin{tabular}{c|c|c|r|c}
\hline \hline Sample & $x_{\mathrm{v}}$ & $\bar{x}$ & \multicolumn{1}{c|}{$\theta[\mathrm{K}]$} & $p\left[10^{21} \mathrm{~cm}^{-3}\right]$ \\
\hline A & 0.095 & 0.020 & 0.706 & 1.04 \\
B & 0.085 & 0.025 & -1.879 & 1.11 \\
C & 0.065 & 0.014 & 0.678 & 1.14 \\
D & 0.045 & 0.009 & 0.373 & 1.49
\end{tabular}

\section{Results and discussion}

The susceptibility data were fitted over the temperature range from 50 to $385 \mathrm{~K}$ to the Curie-Weiss law

$$
\chi=\frac{P_{1}}{T+\theta}+\chi_{0},
$$

where $T$ is the absolute temperature, $P_{1}$ is the Curie constant, $\theta$ is the Curie temperature, and $\chi_{0}$ is the diamagnetic susceptibility of the host lattice. $P_{1}$ and $\theta$ were fitting parameters, $\chi_{0}$ was taken from experimental measurements of $\mathrm{SnTe}$ and varied linearly from $-4.7 \times 10^{-7} \mathrm{emu} / \mathrm{g}$ at $50 \mathrm{~K}$ to $-3.3 \times 10^{-7} \mathrm{emu} / \mathrm{g}$ at $375 \mathrm{~K}$. The effective content of Eu ions, $\bar{x}$, was determined from $P_{1}$ as described in Ref. [3], with estimated error of $20 \%$.

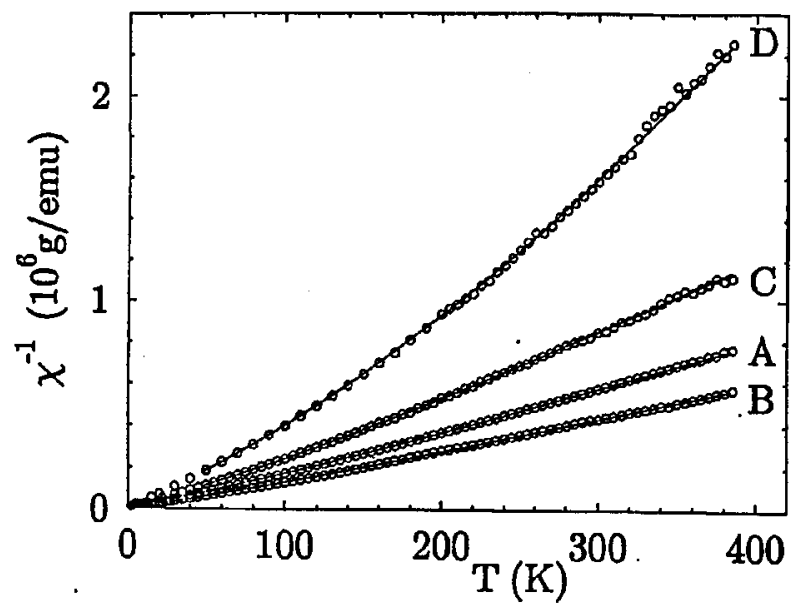

Fig. 1. Inverse susceptibility vs. temperature for $\mathrm{Sn}_{1-x} \mathrm{Eu}_{x} \mathrm{Te}$. Solid lines are fits to the Curie-Weiss law.

The experimental results and fits are shown in Fig. 1. The fitting parameters are given in Table.

There is a large difference between the $x_{\mathrm{v}}$ and $\vec{x}$ parameters. The value $x_{\mathrm{v}}-\bar{x}$ increases with increasing Eu content, followed by a decrease in $p$. It is 
possible that the susceptibility measurements give the number of $\mathrm{Eu}^{2+}$ ions with a spin 3.5, while a large part of $\mathrm{Eu}$ enters the lattice as $\mathrm{Eu}^{3+}$ ions, with no magnetic moment at the ground state, and compensates the $p$-type material.

The Curie temperature, $\theta$, is very small, may be regarded as zero within our experimental accuracy. Therefore, we did not estimate the exchange parameter values from $\theta$.

It was impossible to obtain good fits to the experimental data using a constant diamagnetic susceptibility of SnTe. Therefore, for $\chi_{0}$ we used the exact values obtained from experiment, where $\chi_{0}$ varied linearly with $T$. This approach gave very good fits, as is seen in Fig. 1.

In Fig. 2 we show the magnetic susceptibility vs. temperature at temperatures below $50 \mathrm{~K}$. At about $10 \mathrm{~K}$ there is a cusp in the susceptibility of samples

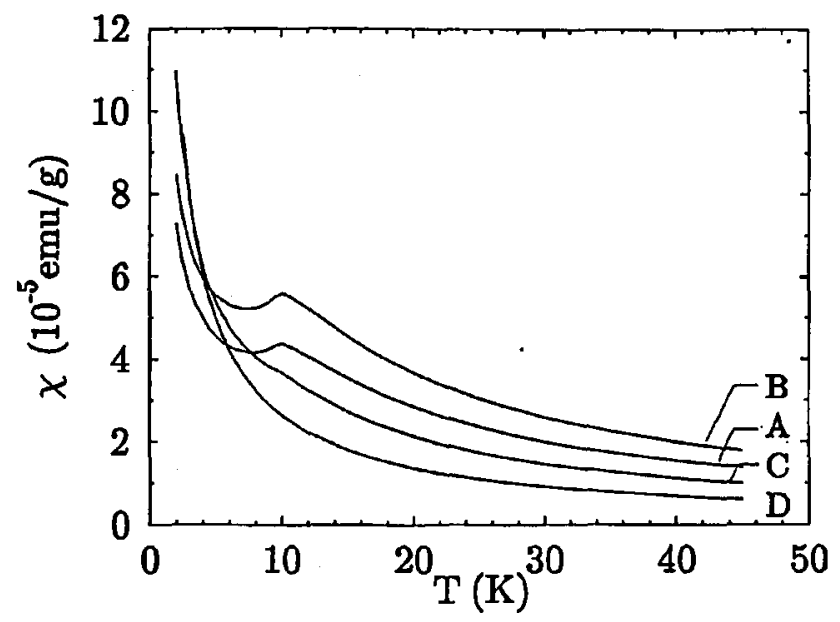

Fig. 2. Magnetic susceptibility vs. temperature for $\mathrm{Sn}_{1-x} \mathrm{Eu}_{x} \mathrm{Te}$. The lines represent the experimental data.

with $x_{\mathrm{v}} \geq 0.085$ and a faint trace of that cusp in the sample with $x_{\mathrm{v}}=0.06 \dot{5}$. That effect, together with the Curie temperature close to zero, indicates a transition to a spin-glass phase, due to antiferromagnetic coupling between Eu ions and ferromagnetic coupling related either to more distant neighbors or carrier-induced RKKY-type interaction, as in $\mathrm{Pb}_{1-x-y} \mathrm{Sn}_{y} \mathrm{Mn}_{x} \mathrm{Te}$ [4].

The subsequent increase in susceptibility below $5 \mathrm{~K}$ may be due to other than Eu paramagnetic impurities or defects. We observed previously similar effect in $p$-type $\mathrm{PbTe}$ [6], explained by Pankratov as related to the magnetic moment of charged nonstoichiometric defects [7].

\section{Acknowledgments}

We are grateful to Mrs. E. Grodzicka for the Hall effect measurements. Thanks are due to the U.S. National Academy of Sciences for the travel support for one of the authors (J.R.A.) to Poland. 


\section{References}

[1] M. Górska, J.R. Anderson, Z. Golacki, Mater. Res. Soc. Symp. Proc. 89, 119 (1987).

[2] M. Górska, J.R. Anderson, G. Kido, Z. Gołacki, Solid State Commun. 75, 363 (1990).

[3] M. Górska, J.R. Anderson, G. Kido, S.M. Green, Z. Gołacki, Phys. Rev. B 45, 11702 (1992).

[4] T. Story, G. Karczewski, L. Świerkowski, R.R. Gałązka, Phys. Rev. B 42, 10477 (1990).

[5] M.P. Mathur, D.W. Weiss; C.K. Jones, A. Patterson, W.J. Carr, Jr., J. Appl. Phys. 42, 1693 (1971).

[6] M. Górska, J.R. Anderson, Acta Phys. Pol. A 75, 273 (1989).

[7] O.A. Pankratov, private information. 